\title{
Phase diagram of the ground states of DNA condensates
}

\author{
Trinh X. Hoang, ${ }^{1}$ Hoa Lan Trinh, ${ }^{1}$ Achille Giacometti, ${ }^{2}$ Rudolf Podgornik,,${ }^{3,4}$ Jayanth R. Banavar, ${ }^{5}$ and Amos Maritan ${ }^{6}$ \\ ${ }^{1}$ Institute of Physics, Vietnam Academy of Science and Technology, 10 Dao Tan, Ba Dinh, Hanoi, Vietnam \\ ${ }^{2}$ Dipartimento di Scienze Molecolari e Nanosistemi, Universita' Ca' Foscari Venezia, I-30123 Venezia, Italy \\ ${ }^{3}$ Department of Theoretical Physics, J. Stefan Institute and Department of Physics, Faculty of Mathematics and Physics, \\ University of Ljubljana, SI-1000 Ljubljana, Slovenia \\ ${ }^{4}$ Department of Physics, University of Massachusetts, Amherst, Massachusetts 01003, USA \\ ${ }^{5}$ Department of Physics, University of Maryland, College Park, Maryland 20742, USA \\ ${ }^{6}$ Dipartimento di Fisica e Astronomia, Università di Padova, CNISM and INFN, via Marzolo 8, I-35131 Padova, Italy
}

(Received 24 June 2015; published 28 December 2015)

\begin{abstract}
The phase diagram of the ground states of DNA in a bad solvent is studied for a semiflexible polymer model with a generalized local elastic bending potential characterized by a nonlinearity parameter $x$ and effective self-attraction promoting compaction. $x=1$ corresponds to the wormlike chain model. Surprisingly, the phase diagram as well as the transition lines between the ground states are found to be a function of $x$. The model provides a simple explanation for the results of prior experimental and computational studies and makes predictions for the specific geometries of the ground states. The results underscore the impact of the form of the microscopic bending energy at macroscopic observable scales.
\end{abstract}

DOI: 10.1103/PhysRevE.92.060701

PACS number(s): 87.14.gk, 87.15.ad, 87.15.Zg

The nonlinear elasticity of DNA at short length scales, probed by nonequilibrium DNA cyclization experiments [1,2], atomic force microscopy (AFM) imaging on surfaces [3], as well as by equilibrium mechanically constrained DNA experiments [4-6], is still not well understood. While the conclusions regarding the first two methods for probing nonlinear doublestranded (ds)-DNA elasticity have been criticized [7], the experiments on stressed DNA ring molecules are performed by a different methodology based on thermodynamic methods via DNA high-curvature states through partial hybridization of a single-stranded (ss)-DNA loop with a linear complementary strand [6]. This methodology does not depend on thermal fluctuations to realize high-curvature states and thus appears to have a far better accuracy and reliability. The nonlinearity in this latter case is clearly present and is captured in a single parameter describing the onset of DNA kink formation. This clearly exhibited elastic nonlinearity is taken as the motivation for the present study that attempts to derive the macroscopic consequences of this interesting microscopic elastic behavior of DNA.

Another interesting facet of DNA behavior is that under specific solution conditions, it condenses into highly compact structures with pronounced symmetry [8-11]. This condensation phenomenon serves as an example of high polymer density packing in biology and of polymer phase transitions and phase separations in general, being relevant also for artificial gene delivery $[12,13]$. Several distinct morphologies of the DNA condensates have been observed including a toroid, a spheroid, as well as a rodlike configuration [10,14-16]. Our principal goal is to explore the rich interplay between the strong tendency for compactness, arising from the presence of multivalent cations or osmolytes in the solution, and the intrinsic stiffness of the DNA molecule promoting the chain to be locally straight. In the absence of stiffness, one would expect the chain to adopt a densely compact spheroidal globule configuration. The key issue is to understand how local stiffness and the detailed way it enters the elastic energy result in the spheroidal configuration becoming unstable with respect to other lower energy configurations. In particular, one would like to map out a phase diagram and understand the different condensate geometries.

The topology of a toroid can be modified in at least two distinct ways. One is to cut and elongate it, making it rodlike, and the other is by filling up the hole. Toroids of sizes larger than $\sim 200 \mathrm{~nm}$ have been observed. In a detailed study [17], the toroid mean diameter was found to vary between 30 and $100 \mathrm{~nm}$ depending on the solution conditions, while the toroid thickness was in the range from 10 to $70 \mathrm{~nm}$. The geometry of a toroid can be characterized by the ratio between its thickness and its diameter. This ratio was typically found to vary for given solution conditions but had a maximum value in a small interval between 0.7 and 0.9 . Ratios close to 1 have been observed for very large toroids [18]. A ratio of 1 corresponds to a toroid with no hole. This is the same topology as a sphere but has different geometry than an isotropic spheroid.

Our study here essentially focuses on the relative stability of different ground states, disregarding the elastic fluctuations around these ground states. An important lesson learned in the study of critical phenomena is that details at the microscopic level often do not matter for the behavior near a critical point. Strikingly, however, we find that simple modifications in the form of the elastic energy penalty at the microscopic scale have considerable macroscopic consequences. The nature of the phase diagram as well as of the phase transitions is found to depend on the details of the local stiffness energy, allowing us to provide a simple explanation for the geometries of the commonly observed toroidal structures of condensed DNA.

The simplest and most commonly used model for describing the stiffness of a chain molecule is the wormlike chain (WLC) model. Consider three (1,2,3) consecutive beads along the chain. Let $\theta$ represent the bending angle, i.e., the angle between vectors $1-2$ and $2-3$. $\theta$ would be zero for a straight segment. The elastic (free) energy penalty on the WLC level is then

$$
u=\kappa(1-\cos \theta)^{x}
$$


where $\kappa$ is the stiffness coefficient per bead and $x=1$. A recent study of the ground states of a chain molecule [19] using the WLC, together with different types of interactions promoting compaction, resulted in a phase diagram with toroidal and rodlike ground states. Furthermore, the combination of analytical calculations and computer simulations showed that the shape of the phase diagram was quite insensitive to the microscopic details. The challenge is then to construct the simplest model, able to qualitatively reproduce the key experimental observations, while at the same time also allowing some flexibility in the predicted behavior, observed in experiments.

A value of $x$ larger than 1 corresponds to a softer potential promoting easy bending. Conversely, the system is stiffer when $x$ is less than 1 . Generally, $x$ cannot be less than $1 / 2$ because of the behavior of the elastic energy for small $\theta$. The coupling between different forms of attractive interaction and thermal fluctuations in WLC polymer collapse was addressed by Hansen et al. [20]. Schnurr et al. [21] employed Brownian dynamics simulations complemented by analytical theory to study the dynamical intermediates for a limiting case of the WLC model. Lappala and Terentjev [22] carried out dynamical computational studies of the compaction of a long chain and observed multiple_-some metastable — configurations. Seaton et al. [23] studied structural phases of semiflexible polymers as a function of temperature and stiffness. The stabilities of toroidal and rodlike condensates under stretching forces have been assessed [24]. Interestingly, DNA-packaging simulations carried out for a $x=1$ chain yield a stable toruslike structure [25]. A careful study by Sakaue and Yoshikawa of a $x=2$ chain dynamics undergoing compaction showed a stable toroidal phase and a metastable rodlike phase [26].

Our analysis of the generalized WLC model, as described by Eq. (1), under compaction shows a rich variety of ground states as a function of the stiffness parameter $x$ : a collapsed spheroid, a rodlike configuration that we model as a cylinder with spherical end caps, a toroid with varying shape ratio, and a swollen extended phase. Surprisingly, the transition lines between these states are found to be characterized by exponents which depend on $x$. In addition, our analysis predicts the geometry of the stable toroids and shows a striking effect of the details of the microscopic bending energy penalty on the macroscopic behavior, relevant for both experiments and simulations.

We model DNA as a semiflexible polymer of length $L=N b$, formed of $N$ spherical beads of diameter $b$. Assume that the morphology of DNA condensate can be either toroidal [Fig. 1(a)] or rodlike [Fig. 1(b)]. The toroidal structure is parametrized by its mean radius $R$ and the thickness radius $\Delta=\alpha R$, with $0<\alpha<1$. The rodlike structure is postulated to have a shape of a spherocylinder of radius $W$ and the cylinder length equal to $\gamma W$ with $\gamma \geqslant 0$. When $\gamma=0$, one obtains the spheroidal configuration [Fig. 1(c)] as a limiting case of the rodlike structure. We will show that the spheroidal configuration will appear as the ground state not only in the limit of $\kappa \rightarrow 0$ or $L \rightarrow \infty$ for $x<3 / 2$, but also as a true ground state when $x>3 / 2$.

Assume a close-packed hexagonal chain packing in the condensate [10] with DNA-DNA interaxial spacing $d$. The DNA volume packing fraction is $\eta=(\pi / \sqrt{12})(b / d)^{2}$. The toroidal (a)

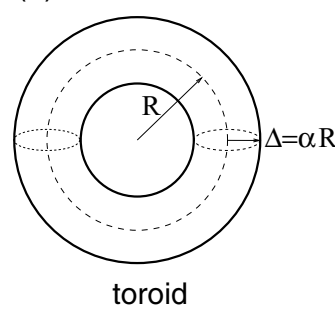

toroid

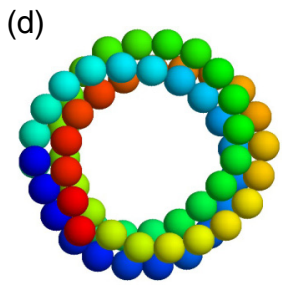

$x=1, \kappa=12$ (b)

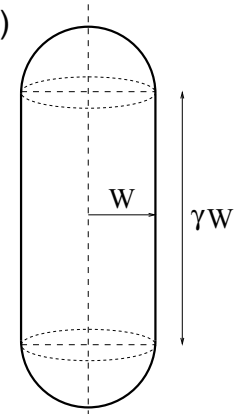

rod-like

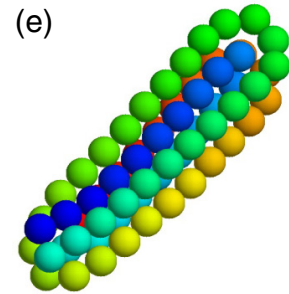

$x=1, \kappa=8$ (c)

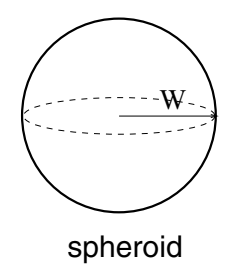

(f)

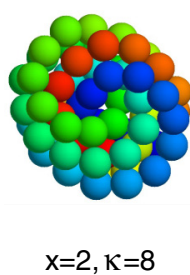

FIG. 1. (Color online) Schematics of (a) toroidal, (b) rodlike, and (c) spheroidal models of DNA condensates. Ground state conformations obtained in Monte Carlo simulations of a $N=64$ bead-and-spring model with $x=1$ generalized elastic potential at (d) moderate and (e) low stiffness and $x=2$ at (f) low stiffness. The conformation (f) is a torus with no hole.

mean radius can be expressed as $R=\frac{1}{2}\left(L b^{2} / \pi \eta\right)^{1 / 3} \alpha^{-2 / 3}$, while the thickness radius of the rodlike condensate is $W=$ $\left(3 L b^{2} / 4 \eta\right)^{1 / 3}(4+3 \gamma)^{-1 / 3}$. For the toroidal condensate, we assume that the chain has a constant radius of curvature [27] equal to its mean radius $R$. The alternative "spool" model [28] for chain wrapping does not change the characteristics of the phase diagram and yields a higher bending energy than the constant curvature model. For the rodlike condensate, we assume that the radius of curvature is uniformly equal to $W / 2$ in the spherical caps of the spherocylinder, whereas the curvature is zero in the cylinder body.

The chain compaction is induced by introducing a surface term in the total energy of the condensate, with $\sigma>0$ a surface energy per unit area. For the toroidal condensate, the total energy including the bending energy is then

$$
E_{\mathrm{tor}}=\kappa \frac{L}{b}\left(\frac{b^{2}}{2 R^{2}}\right)^{x}+\sigma 4 \pi^{2} \alpha R^{2},
$$

and for the rodlike condensate, respectively,

$$
E_{\text {rod }}=\frac{2^{4+x}}{3} \kappa \eta\left(\frac{b}{W}\right)^{2 x-3}+\sigma 2 \pi(2+\gamma) W^{2} .
$$

For a given $L$ and $\kappa$, the minimum energy $E_{\text {tor }}^{*}$ and the optimal ratio $\alpha^{*}$ for the toroidal condensate are obtained by minimizing $E$ with respect to $\alpha$. The rodlike condensate can be a ground state only if $x<\frac{3}{2}$, when $\partial E_{\text {rod }} / \partial \gamma=0$ has a positive root, $\gamma^{*}>0$. For $x>\frac{3}{2}$, there are no positive roots, and the minimum energy is the spheroidal configuration $\left(\gamma^{*}=0\right)$. 


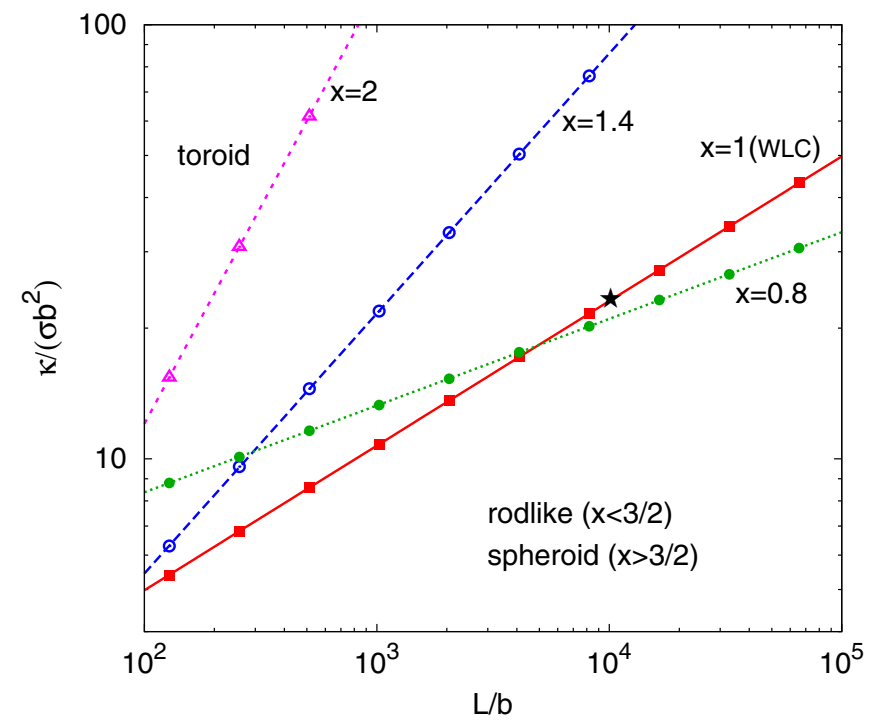

FIG. 2. (Color online) Ground state phase diagrams in the lengthstiffness plane of a semiflexible chain with the generalized elastic potential for several values of $x$, as indicated. The lines correspond to transitions from a toroidal to a rodlike condensate for $x<3 / 2$, and from a toroidal to a spheroidal configuration for $x>3 / 2$, being fits with slopes equal to $\frac{2 x-1}{3}$ to numerical data points. An experimental data point (star) shown on the WLC model transition line corresponds to the average DNA length of $\sim 63.6 \mathrm{kbp}$ in $90 \mathrm{~nm}$ sized condensates found for a plasmid DNA condensation induced by cobalt(III) hexammine $\left(\mathrm{CoHex}^{3+}\right)$ with a coexistence of toroid phase and rodlike phase [14]. Projection from this point on the vertical axis yields $\sigma \approx 0.266 k_{B} T(\mathrm{~nm})^{-2}$, or equivalently, an intermolecular contact interaction energy of $\varepsilon \approx-\sigma d \approx-0.246 k_{B} T / \mathrm{bp}$, by using the DNA persistence length $\beta \kappa b=50 \mathrm{~nm}$, diameter $b=2 \mathrm{~nm}$, and the interaxial spacing $d=2.8 \mathrm{~nm}$. The energy $\varepsilon$ roughly agrees with that for the $\mathrm{CoHex}^{3+}$ mediated DNA-DNA interaction obtained by the osmotic stress method $\left(-0.21 \pm 0.02 k_{B} T / \mathrm{bp}\right)$ [29].

By comparing $E_{\text {tor }}^{*}$ with $E_{\text {rod }}^{*}$, we constructed the phase diagrams of the ground states in the $\kappa-L$ plane (Fig. 2). For a given $L$, one observes a transition from rodlike or spheroidal to the toroidal condensate on increasing $\kappa$. For very large stiffness, there is another transition from the toroidal to an open straight conformation (not shown in Fig. 2). It can be shown that the ratio $E_{\text {rod }}^{*} / E_{\text {tor }}^{*}$ is a function of $\sigma / \kappa L^{(2 x-1) / 3}$, so the only way to make it equal to 1 while changing $L$ and $\kappa$ is to have $\sigma / \kappa L^{(2 x-1) / 3}=$ const. Thus, at the transition line between the toroidal and the rodlike condensate, one has

$$
\frac{\kappa}{\sigma} \sim L^{\frac{2 x-1}{3}}
$$

For $x=1$, one recovers the power law of $L^{1 / 3}$ [19]. For $x=$ 0.5 , the transition line would become independent of $L$, but for this limiting case, both the toroid phase and the rodlike phase disappear (see below).

Coexisting toroidal and rodlike condensates of similar volume and dimensions have been observed in experiments $[14,15]$, suggesting that those condensates were close to a phase transition between the two kinds of structures. Figure 2 shows that fitting the experimental data to the phase diagram yields a reasonable energy for a DNA-DNA interaction. The
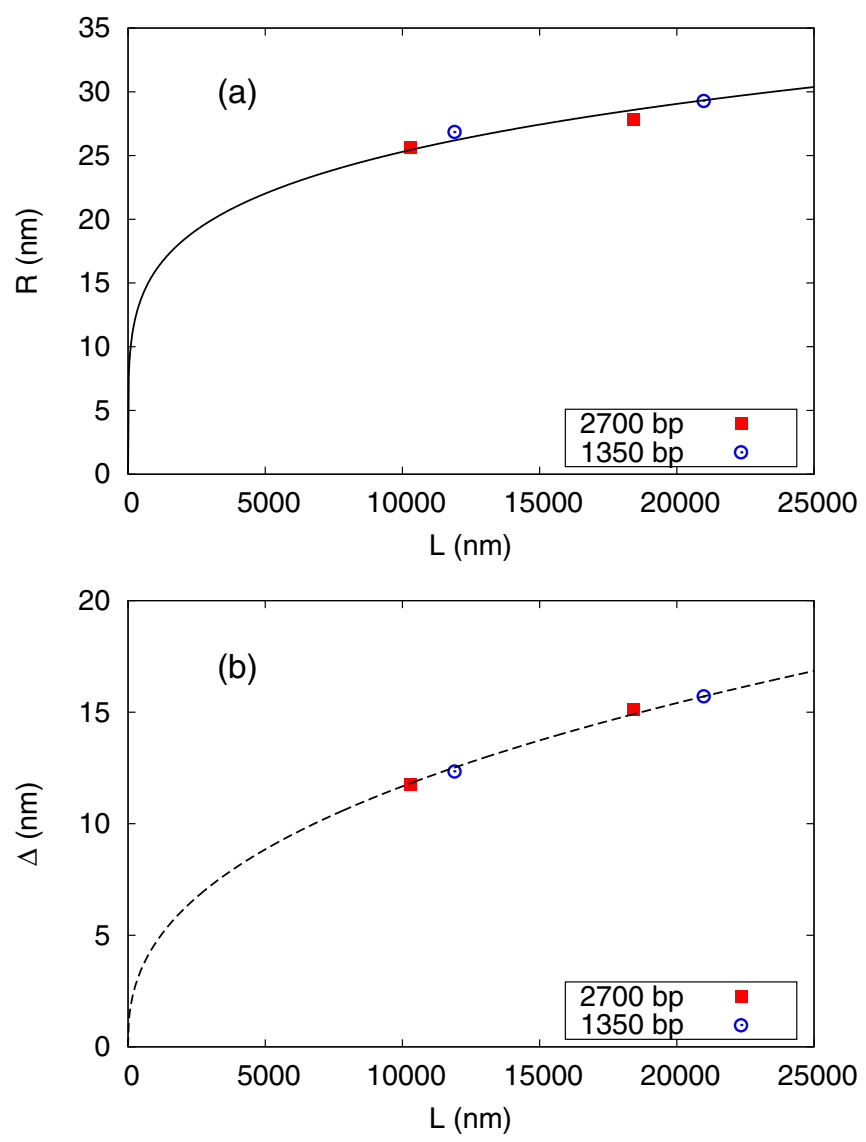

FIG. 3. (Color online) Dependence of the toroidal mean radius $R$ and the toroidal thickness radius $\Delta$ on the total contour length $L$ of DNA in toroidal condensates. Experimental data points are fitted to the theoretical results for the WLC model $(x=1)$ as $R \sim L^{1 / 5}$ (solid line) and $\Delta \sim L^{2 / 5}$ (dashed line) in (a) and (b), respectively. Experimental data are taken from Ref. [14] for condensation of plasmid DNA fragments of lengths $2700 \mathrm{bp}$ (squares) and $1350 \mathrm{bp}$ (circles) with [DNA] $=10 \mu \mathrm{g} / \mathrm{mL}$. The condensation was induced by $150 \mu \mathrm{M} \mathrm{CoHex}{ }^{3+}$ and the sizes of the condensates were measured at $2 \mathrm{~h}$ and at $24 \mathrm{~h}$ after polyvalent salt addition. The volume of an average toroid was found to increase by $\sim 50 \%$, between 2 and 24 h. $R$ and $\Delta$ are calculated based on the measured average toroid outer radius $R_{1}$ and inner radius $R_{2}$ given in the captions of Figs. 3 and 4 of Ref. [14] as $R=\left(R_{1}+R_{2}\right) / 2$ and $\Delta=\left(R_{1}-R_{2}\right) / 2$, respectively. The DNA contour length in a condensate is determined as $L=8 \pi \eta R(\Delta / b)^{2}$ by assuming a hexagonal close packing of DNA in the condensate with the volume fraction $\eta=(\pi / \sqrt{12})(b / d)^{2}$, where $d=2.8 \mathrm{~nm}$ is the DNA-DNA interaxial spacing, and $b=2 \mathrm{~nm}$ is the diameter of DNA.

relatively larger volume seen for spheroidal condensates with respect to toroidal ones, as observed in T4 DNA condensation using protamine sulfate as the condensing agent [16], agrees with our phase diagram. The observation of the spheroidal condensates also suggests that the highly charged protamines, when bound to DNA major grooves [30], strongly modify the DNA elasticity making it incompatible with the WLC model. Our generalized elasticity model is applicable also to multimolecular condensates, since due to a strong sticky hydrophobic interaction between the exposed ends of DNA 

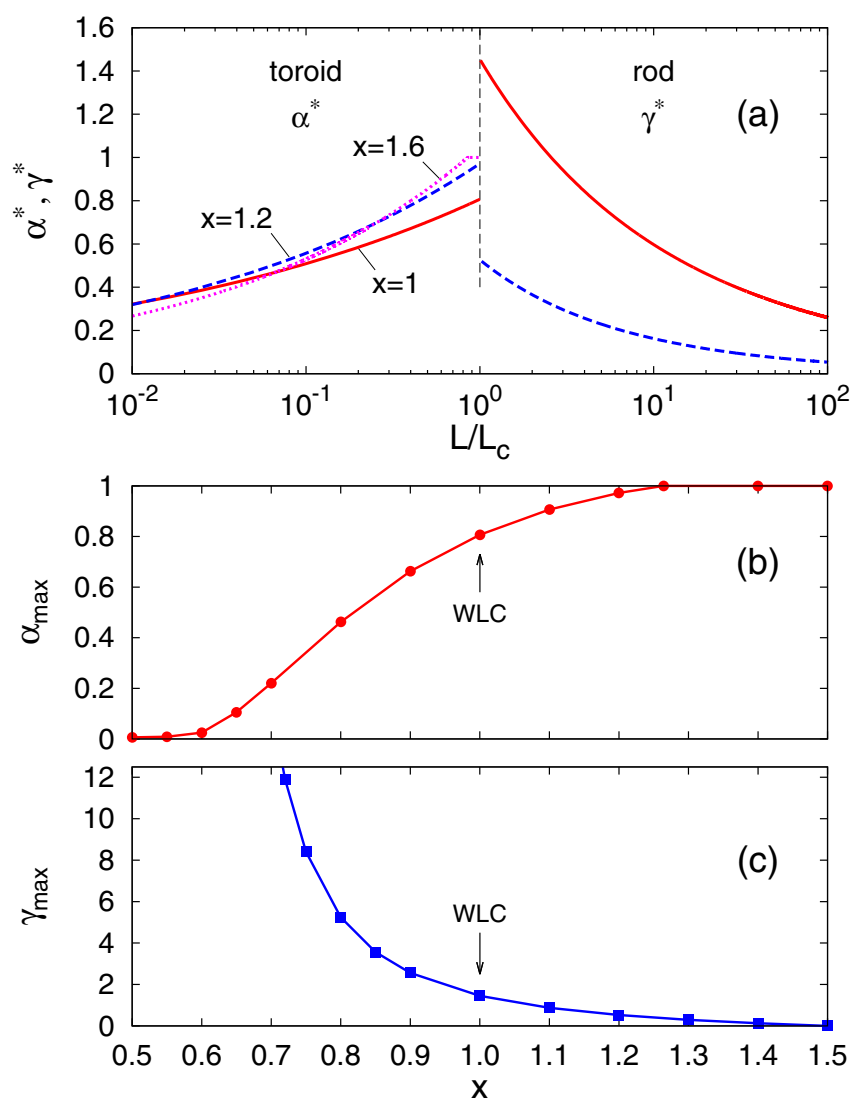

FIG. 4. (Color online) (a) Dependence of geometrical parameters $\alpha$ and $\gamma$ on the chain length $L$ for toroidal (left) and rodlike (right) condensates. At $L=L_{c}$ the chain undergoes a transition from a toroidal to rodlike conformation. The data shown are obtained for $x=1$ (solid), $x=1.2$ (dashed), and $x=1.6$ (dotted), as indicated. The dependence of the maximum values $\alpha_{\max }$ and $\gamma_{\max }$ of the parameters $\alpha$ and $\gamma$ on the exponent $x$ are shown in (b) and (c), respectively. The arrow indicates the result for the WLC model $(x=1)$, for which $\alpha_{\max } \approx 0.808$ and $\gamma_{\max } \approx 1.45$. $\alpha_{\max }$ increases from zero at $x=0.5$ and reaches unity at $x \approx 1.264$, whereas $\gamma_{\max }$ becomes zero only at $x=1.5$.

fragments, there is no significant free energy contribution from linear aggregation of the molecules [31].

Interestingly, the geometrical parameters $\alpha^{*}$ and $\gamma^{*}$ are also found to be functions of $\sigma / \kappa L^{(2 x-1) / 3}$, and thus are constant along the transition line for a given $x$. For both types of condensates, the size can be normalized by $L_{c}$, the length of the polymer at the transition line. For toroidal condensates it then follows $\alpha^{*} \sim\left(L / L_{c}\right)^{\frac{2 x-1}{4 x+1}}$ when $\alpha^{*}<1$, whereas for the rodlike condensates, the dependence of $\gamma^{*}$ on $L$ is not trivial. For large lengths $\left(L \gg L_{c}\right)$, we remain with $\gamma^{*} \sim\left(L / L_{c}\right)^{\frac{1-2 x}{3}}$. It is also straightforward to show that the toroid mean radius $R$ depends on the chain length as $R \sim L^{\frac{1}{4 x+1}}$, and the toroid's thickness scales with its mean radius as $\Delta \sim R^{2 x}$. For $x=1$ one finds that $\Delta \sim R^{2}$, meaning that a big toroid is also much thicker than a small toroid, as commonly observed in experiments. Figure 3 shows that the scaling of $R$ and $\Delta$ with $L$ also

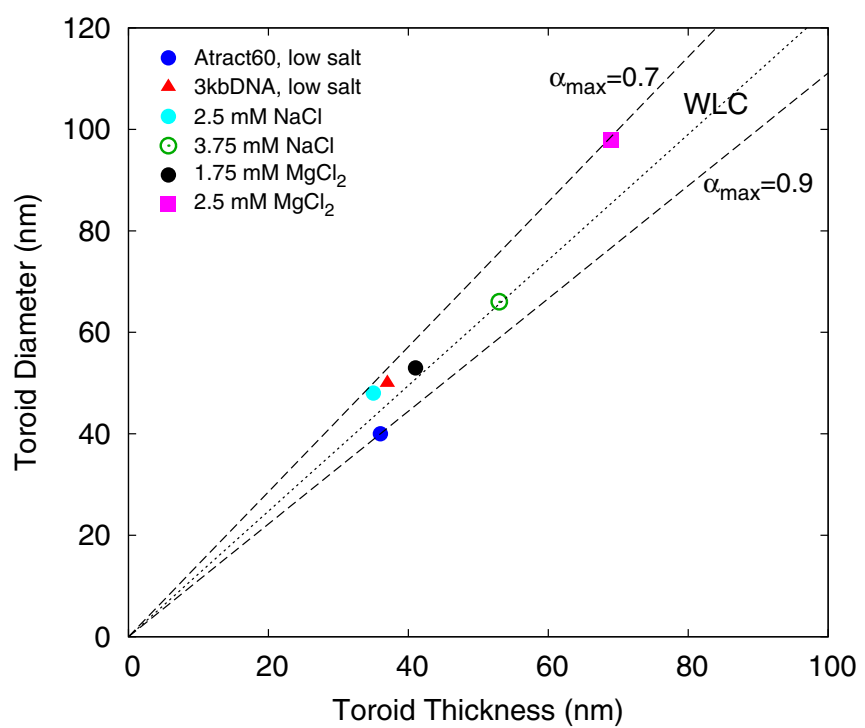

FIG. 5. (Color online) Toroid thickness vs toroid diameter as obtained by experiments at various conditions (data points). The data points were extracted from Fig. 2 of Ref. [17]. For a given condensation condition (see legend), we take only the point with the maximum thickness to diameter ratio. The lines show the slopes corresponding to the maximum aspect ratios $\alpha_{\max }$ as predicted from our model on changing the parameter $x$. For the wormlike chain (WLC), $\alpha_{\max }=0.808$ (dotted line).

agrees with available experimental data for the condensation of plasmid DNA [14].

Figure 4(a) shows the dependence of $\alpha^{*}$ and $\gamma^{*}$ on $L$ away from the transition line. At $L=L_{c}$, both $\alpha^{*}$ and $\gamma^{*}$ have maximum values $\alpha_{\max }$ and $\gamma_{\max }$, respectively, that strongly depend on $x$. For a given stiffness on an increase of $L$ the toroid condensate becomes fatter and attains its maximum thickness at the transition line. For $x>1.246$ the toroid then attains its maximum thickness with the ratio $\alpha=1$ even before $L$ reaches $L_{c}$ - the doughnut has no hole [see the curve for $x=1.6$ in Fig. 4(a)]. As $L$ becomes larger than $L_{c}$, the rodlike condensate becomes the ground state, and eventually its length to thickness ratio $\gamma^{*}$ starts decreasing. As $L \rightarrow \infty, \gamma^{*}$ becomes zero and the rodlike condensate turns into a globule.

At the transition line, both $L$ and $\kappa$ can be renormalized and the maximum values $\alpha_{\max }$ and $\gamma_{\max }$ become functions of the parameter $x$ only. Figures 4(b) and 4(c) show the dependence of $\alpha_{\max }$ and $\gamma_{\max }$ on $x$, for $x \in[0.5,1.5]$. For $x=0.5, \alpha_{\max }=0$, while $\gamma_{\max }$ diverges, indicating that both the toroidal and the rodlike phases disappear in this limit. $\alpha_{\max }$ increases with $x$ to unity at $x \approx 1.246$, while $\gamma_{\max }$ decreases to zero as $x$ approaches 1.5. This again confirms that for $x \geqslant 1.5$ the rodlike condensate becomes a globule for all lengths. For the WLC model $(x=1), \alpha_{\max } \approx 0.808$ and $\gamma_{\max } \approx 1.45$.

It has been suggested that deviations from WLC occur at length scales less than $100 \mathrm{~nm}$ [3]. In Fig. 5, we show some experimental data for the maximum thickness to diameter ratio (data extracted from Ref. [17]) under various solution conditions. Experimental points correspond to $\alpha_{\max }$ varying between 0.7 and 0.9 corresponding to $x$ in the range from 0.9 to 1.1. Thus, our analysis suggests that the solution conditions 
may be captured through the exponent $x$ in the generalized elastic potential of DNA inside the condensate, but the bending elasticity remains essentially that of the WLC, i.e., $x \approx 1 \pm 0.1$. This suggests that DNA compaction does not involve high deformation kinking of the chain, consistent with various experimental studies on the looping of DNA (see, e.g., Ref. [32]), which indicated that the wormlike chain model is a reasonable model for the elastic nature of DNA at short lengths.

This research is funded by Vietnam National Foundation for Science and Technology Development (NAFOSTED) under Grant No. 103.01-2013.16. A.G. acknowledges funding from PRIN-COFIN2010-2011 (Contract No. 2010LKE4CC).
[1] T. E. Cloutier and J. Widom, Mol. Cell. 14, 355 (2004).

[2] Q. Du, C. Smith, N. Shiffeldrim, M. Vologodskaia, and A. Vologodskii, Proc. Natl. Acad. Sci. USA 102, 5397 (2005).

[3] P. A. Wiggins, T. van der Heijden, F. Moreno-Herrero, A. Spakowitz, R. Phillips, J. Widom, C. Dekker, and P. C. Nelson, Nat. Nanotechnol. 1, 137 (2006).

[4] H. Qu, C.-Y. Tseng, Y. Wang, A. J. Levine, and G. Zocchi, Europhys. Lett. 90, 18003 (2010).

[5] H. Qu and G. Zocchi, Europhys. Lett. 94, 18003 (2011).

[6] H. Qu, Y. Wang, C.-Y. Tseng, and G. Zocchi, Phys. Rev. X 1, 021008 (2011).

[7] A. Vologodski and M. D. Franl-Kamenetskii, Nucleic Acids Res. 41, 6785 (2013).

[8] L. C. Gosule and J. A. Schellman, Nature (London) 259, 333 (1976).

[9] V. A. Bloomfield, Curr. Opin. Struct. Biol. 6, 334 (1996).

[10] N. V. Hud and I. D. Vilfan, Annu. Rev. Biophys. Biomol. Struct. 34, 295 (2005).

[11] H. H. Strey, R. Podgornik, D. D. Rau, and V. A. Parsegian, Curr. Opin. Struct. Biol. 8, 309 (1998).

[12] A. Leforestier, A. Šiber, F. Livolant, and R. Podgornik, Biophys. J. 100, 2209 (2011).

[13] R. Podgornik, D. Harries, J. DeRouchey, H. H. Strey, and V. A. Parsegian, in Gene Therapy: Therapeutic Mechanisms and Strategies, edited by N. S. Templeton (Dekker, New York, 2008), 3rd ed.

[14] P. G. Arscott, A.-Z. Li, and V. A. Bloomfield, Biopolymers 30, 619 (1990).

[15] R. Golan, L. I. Pietrasanta, W. Hsieh, and H. G. Hansma, Biochemistry 38, 14069 (1999).

[16] M. F. V. Pinto, M. C. Moran, M. G. Miguel, B. Lindman, A. S. Jurado, and A. A. C. C. Pais, Biomacromolecules 10, 1319 (2009).
[17] C. C. Conwell, I. D. Vilfan, and N. V. Hud, Proc. Natl. Acad. Sci. USA 100, 9296 (2003).

[18] C. C. Conwell and N. V. Hud, Biochemistry 43, 5380 (2004).

[19] T. X. Hoang, A. Giacometti, R. Podgornik, N. T. T. Nguyen, J. R. Banavar, and A. Maritan, J. Chem. Phys. 140, 064902 (2014).

[20] P. L. Hansen, D. Svensek, V. A. Parsegian, and R. Podgornik, Phys. Rev. E 60, 1956 (1999).

[21] F. Schnurr, F. C. MacKintosh, and D. R. M. Williams, Europhys. Lett. 51, 279 (2000).

[22] A. Lappala and E. M. Terentjev, Macromolecules 46, 7125 (2013).

[23] D. T. Seaton, S. Schnabel, D. P. Landau, and M. Bachmann, Phys. Rev. Lett. 110, 028103 (2013).

[24] R. Cortini, B. R. Care, J.-M. Victor, and M. Barbi, J. Chem. Phys. 142, 105102 (2015).

[25] D. Marenduzzo, E. Orlandini, A. Stasiak, D. W. Sumners, L. Tubiana, and C. Micheletti, Proc. Natl. Acad. Sci. USA 106, 22269 (2009).

[26] T. Sakaue and K. Yoshikawa, J. Chem. Phys. 117, 6323 (2002).

[27] N. V. Hud, K. H. Downing, and R. Balhorn, Proc. Natl. Acad. Sci. USA 92, 3581 (1995).

[28] J. Widom and R. L. Baldwin, J. Mol. Biol. 171, 419 (1983).

[29] B. A. Todd, V. A. Parsegian, A. Shirahata, T. J. Thomas, and D. C. Rau, Biophys. J. 94, 4775 (2008).

[30] R. Balhorn, Genome Biol. 8, 227 (2007).

[31] G. Zanchetta, F. Giavazzi, M. Nakata, M. Buscaglia, R. Cerbino, N. A. Clark, and T. Bellini, Proc. Natl. Acad. Sci. USA 107, 17497 (2010).

[32] Y. J. Chen, S. Johnson, P. Mulligan, A. J. Spakowitz, and R. Phillips, Proc. Natl. Acad. Sci. USA 111, 17396 (2014). 